\title{
Digital synthesis of pulse shapes in real time for high resolution radiation spectroscopy
}

\author{
Valentin T. Jordanov *, Glenn F. Knoll \\ Department of Nuclear Engineering, The Universtty of Michtgan, 2355 Bonisteel Blvd., Ann Arbor, MI 48109, USA
}

(Received 18 January 1994)

Techniques have been developed for the synthesis of pulse shapes using fast digital schemes in place of the traditional analog methods of pulse shaping. Efficient recursive algorithms have been developed that allow real time implementation of a shaper that can produce either trapezoidal or triangular pulse shapes. Other recursive techniques are presented which allow a synthesis of finite cusp-like shapes. Preliminary experimental tests show potential advantages of using these techniques in high resolution, high count rate pulse spectroscopy.

\section{Introduction}

The theory of optimal pulse shaping in high resolution spectroscopy has been well developed and published in number of articles and textbooks. While the ideal cusp shape has been cited to give the best signalto-noise ratio, it has been recognized that this pulse shape is not practical because of its theoretically infinite time duration $[1,2]$. In a typical spectroscopy configuration employing a charge sensitive preamplifier, two generalized types of noise are of significant importance: step and delta noise [1,3]. When delta noise is predominant, the optimum pulse shape for a finite pulse duration is the symmetrical triangular shape $[1,4]$. In addition to electronic noise, the pulse height measurement is subject to other sources of fluctuations such as statistical variations in the number of charge carriers, microphonic effects, charge loss due to trapping, etc. [5]. An additional consideration is the sensitivity of the pulse shaping techniques to variations in the detector charge collection time. Ballistic deficit and charge trapping effects can noticeably reduce the resolution of spectrometers when shapers producing short pulses with sharp peaks are used. It has been demonstrated that these effects can be reduced by using flat-topped shapes $[1,6,7]$

This paper describes a technique for synthesis of optimal pulse shapes (symmetric triangle and symmetric trapezoid) for high resolution, high throughput spectroscopy, using fast recursive digital algorithms.

\footnotetext{
* Corresponding author. Present address: Amptek Inc., $6 \mathrm{De}$ Angelo Dr., Bedford, MA 01730, USA.
}

The technique also allows synthesis of other shapes such as finite cusp-like and flat-topped cusp-like shapes. The algorithms are suitable for real time implementation, require only simple hardware, and offer flexibility in the adjustment of the output pulse shapes.

\section{Synthesis of trapezoidal and triangular pulses}

In common spectroscopy systems, the detector-preamplifier configuration is followed by a prefilter circuit (pole-zero cancellation, CR differentiation) that produces a pulse with short rise time followed by a long exponential tail. In the following development, we assume this type of input signal with amplitude normalized to one and a decay time constant of $\tau$. The rise time of the pulse, for now, will be considered to be very short. An example of the exponential signal is sketched in Fig. 1a.

The initial problem we have addressed is to develop an efficient algorithm to convert this exponential pulse to a pulse with true trapezoidal shape. We will first carry on the analysis in the continuous time domain, and then apply the results in the discrete time domain.

The output $s(t)$ of a linear time-invariant system is given by the convolution integral

$s(t)=\int_{-\infty}^{+\infty} \nu\left(t^{\prime}\right) h\left(t-t^{\prime}\right) \mathrm{d} t^{\prime}$,

where $\nu(t)$ is the input signal and $h(t)$ is the impulse response of the system. For real-time signal processing the system must be causal, i.e. the output at given moment depends only on present and previous values of the input signal. As a result the upper limit of 
a)

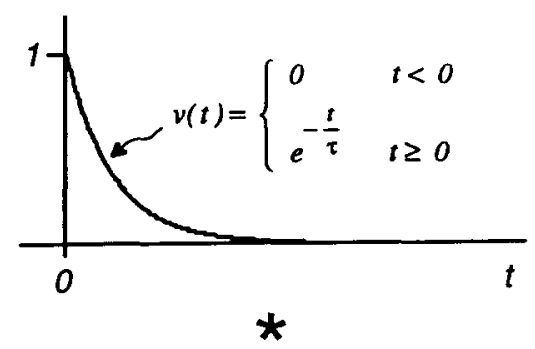

b)

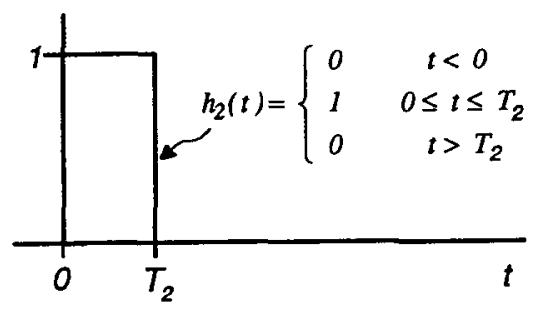

c)

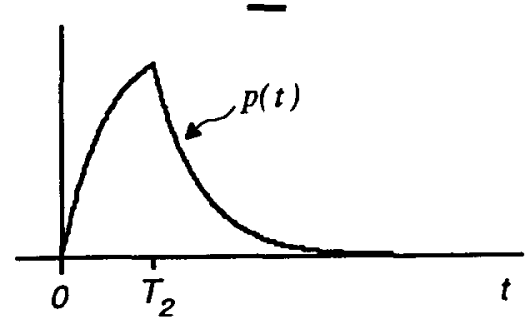

Fig. 1. Convolution of an exponential input signal (a) with rectangular function (b) and the output response of the system $(c)$

integration in Eq. (1) can be truncated at the moment of time $t$ at which the output of the system is evaluated. Our goal is to find a causal system with finite impulse response which, when used in Eq. (1), will transform an exponential input signal into a trapezoidal shape. The convolving function should also be simple and practically realizable.

\subsection{Convolution with rectangular and truncated ramp functions}

First consider a system with a simple rectangular impulse response, also called a moving average system. The digital implementation of this system in radiation spectrometry has been previously described [8]. An example of a unit rectangular function is shown in Fig. $1 b$. The output response of the moving average system to an exponential input signal (Fig. 1a) is presented in Fig. 1c. The result of the convolution of an exponential signal with a unit rectangular function is described by the following equations

$p(t)=\int_{0}^{t} \mathrm{e}^{\left(t^{\prime}-t\right) / \tau} \mathrm{d} t^{\prime}=\tau\left(l-\mathrm{e}^{-t / \tau}\right), \quad 0 \leq t \leq T_{2}$ and

$p(t)=\int_{0}^{T_{2}} \mathrm{e}^{\left(t^{\prime}-t\right) / \tau} \mathrm{d} t^{\prime}=\tau \mathrm{e}^{-t / \tau}\left(\mathrm{e}^{T_{2} / \tau}-1\right), \quad t>T_{2}$.

The response of the system for $t<0$ is zero. Note that the only time dependent terms in Eq. (2) and Eq. (3) are exponentials. An important feature of the output signal is that, after reaching a maximum, it decays with the same time constant as the time constant of the input signal.

Another simple convolving function is the truncated ramp (or saw-tooth) function. Fig. 2b shows a unit slope truncated ramp function with duration $T_{1}$. The response of this system to an exponential signal (Fig. 2a) is depicted in Fig. 2c. The output response of this system is given by

$$
\begin{aligned}
& r(t)=\int_{0}^{t} t^{\prime} \mathrm{e}^{\left(t^{\prime}-t\right) / \tau} \mathrm{d} t^{\prime}=\tau t-\tau^{2}\left(1-\mathrm{e}^{-t / \tau}\right), \\
& 0 \leq t \leq T_{1}
\end{aligned}
$$

a)

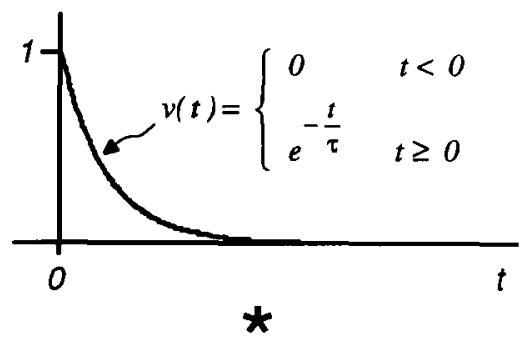

b)

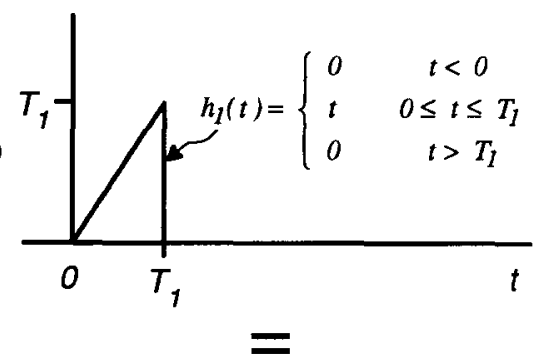

c)

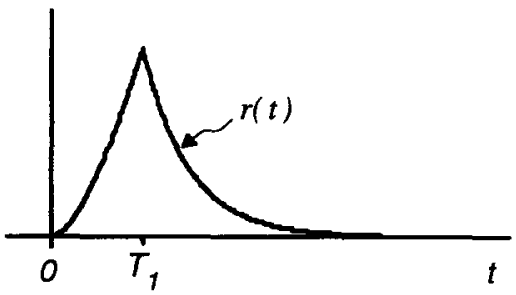

Fig. 2. Convolution of an exponential input signal (a) with unit slope truncated ramp function (b) and the response of the system (c). 
and

$$
\begin{aligned}
r(t) & =\int_{0}^{T_{1}} t^{\prime} \mathrm{e}^{\left(t^{\prime}-t\right) / \tau} \mathrm{d} t^{\prime} \\
& =\tau \mathrm{e}^{-t / \tau}\left(\tau+\mathrm{e}^{T_{1} / \tau}\left(T_{1}-\tau\right)\right), \quad t>T_{1} .
\end{aligned}
$$

Again the response of this system for $t<0$ is zero, and this case will be omitted in the following discussions. Eq. (4) has two time dependent terms - one is linear and the other is exponential.

\subsection{Impulse response of the trapezoidal shaper}

We next will develop the conditions under which a flat-topped trapezoidal shape can be synthesized. Taking the definitions of $r(t)$ and $p(t)$ from above, consider a function $f(t)$ defined as

$f(t)=r(t)+\tau p(t)+a p\left(t-T_{1}\right)$,

where $a$ is a parameter. It is assumed also that $T_{1} \leq T_{2}$. Further, for simplification, the function describing the output signal of the system in given time interval will be indicated using two indices referring to the beginning and end of the interval, e.g. $f_{01}(t)$ denotes $f(t)$ in the time interval $0 \leq t<T_{1}, f_{12}(t)$ implies $f(t)$ for $T_{1} \leq t<T_{2}$, etc. Using this convention and defining a moment of time $T_{3}=T_{2}+T_{1}$, Eq. (6) can be rewritten as three separate equations

$$
\begin{aligned}
f_{01}(t)= & \tau t \\
f_{12}(t)= & \tau^{2}\left(1-\mathrm{e}^{-t / \tau}\right)+\tau \mathrm{e}^{-t / \tau}\left(\tau+\mathrm{e}^{T_{1} / \tau}\left(T_{1}-\tau\right)\right) \\
& +a \tau\left(1-\mathrm{e}^{-\left(t-T_{1}\right) / \tau}\right) \\
= & \tau^{2}+a \tau+\tau \mathrm{e}^{-\left(t-T_{1}\right) / \tau}\left(T_{1}-\tau-a\right) \\
f_{23}(t)= & \tau^{2} \mathrm{e}^{-t / \tau}\left(\mathrm{e}^{T_{2} / \tau}-1\right) \\
& +\tau \mathrm{e}^{-t / \tau}\left(\tau+\mathrm{e}^{T_{1} / \tau}\left(T_{1}-\tau\right)\right) \\
& +a \tau\left(1-\mathrm{e}^{-\left(t-T_{1}\right) / \tau}\right) .
\end{aligned}
$$

Eq. (7) represents the linear rising slope of the desired trapezoidal shape. The next step is to find the condition under which $f(t)$ is constant in the interval $T_{1} \leq t$ $\leq T_{2}$. This condition can be determined from Eq. (8). Since $f_{12}(t)$ must be time independent, it is necessary that $T_{1}-\tau-a=0$ or $a=T_{1}-\tau$. For the case in which $a=T_{1}-\tau$, Eq. (8) and Eq. (9) reduce to

$f_{12}(t)=T_{1} \tau$,

$f_{23}(t)=\tau^{2} \mathrm{e}^{-\left(t-T_{2}\right) / \tau}+\tau\left(T_{1}-\tau\right)$.

This choice of $a=T_{1}-\tau$ is assumed for the remainder of this analysis. If the trapezoidal shape is to be symmetric, the signal must decay to zero at $T_{3}=T_{2}+T_{1}$. Let the response of the system be written as

$s(t)=f(t)+q(t)$ where $q(t)$ is the specific function that results in $s(t)$ becoming a symmetric trapezoidal function defined as

$s_{-\infty 0}(t)=0$,

$s_{01}(t)=\tau t$,

$s_{12}(t)=T_{1} \tau$,

$s_{23}(t)=T_{1} \tau-\tau\left(t-T_{2}\right)$,

$s_{3 \circ}(t)=0$.

The specific solution for $q(t)$ can be found from Eq. (7) and Eq. (10) through 17 . The result is that $q(t)=0$ in the time interval $-\infty<t<T_{2}$, and in the rest of the time domain $q(t)$ is defined by

$q_{23}(t)=-\left[\tau\left(t-T_{2}\right)-\tau^{2}\left(1-\mathrm{e}^{-\left(t-T_{2}\right) / \tau}\right)\right]$

and

$q_{3 \circ 0}(t)=-\left[\tau^{2} \mathrm{e}^{-\left(t-T_{2}\right) / \tau}\right]$

Eqs. (4), (5), (18) and (19) show that $q(t)$ has the same shape as $r(t)$ but with opposite polarity and a delay in time of $T_{2}$

$q(t)=-r\left(t-T_{2}\right)$.

Thus, a system with properties that transform an exponential to a trapezoidal shape has been determined. In the case of $T_{1}=T_{2}$ the trapezoid is reduced to a triangular shape. It should be noted that other constraints can be applied regarding the shape and duration of trailing edge of the trapezoidal pulse, and asymmetric shapes can be obtained. However, a symmetric shape is generally preferred for optimum signalto-noise ratio in the measurement of pulse height [3].

From the distributive property of convolutions, the impulse response of the system can be written as

$$
\begin{aligned}
h(t)= & h_{1}(t)+\tau h_{2}(t)+\left(T_{1}-\tau\right) h_{2}\left(t-T_{1}\right) \\
& -h_{1}\left(t-T_{2}\right),
\end{aligned}
$$

where $h_{1}(t)$ and $h_{2}(t)$ are the impulse responses of the truncated ramp system and the moving average system respectively. The impulse response of the trapezoidal shaping system is shown in Fig. 3b. In this example $T_{1}=\tau$. This response is synthesized by simple functions and is suitable for real time digital implementation.

\section{Digital implementation of the trapezoidal shaper}

Consider an exponentially-decaying input signal that is digitally sampled at equal intervals of time. In what follows, we will measure time in units of the sampling period. The input signal at time $i$ will be written as $\nu(i)$. The first step in the synthesis is to convolve the sampled input signal with a rectangular function. Since it is required that the convolution be performed in real 
a)

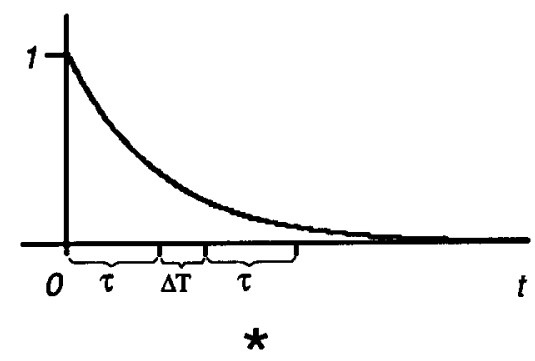

b)

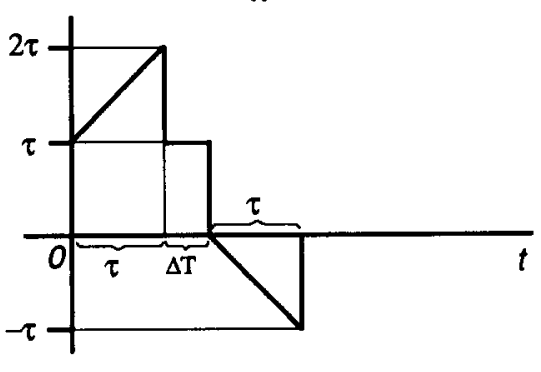

c)

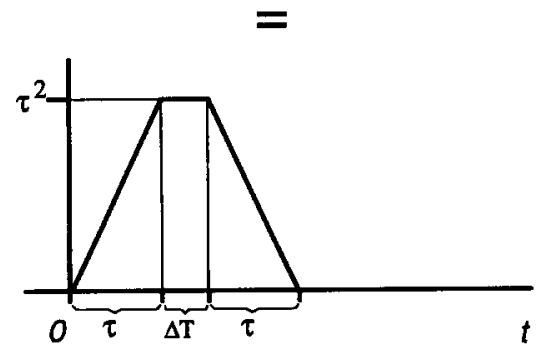

Fig. 3. Convolution of an exponential input signal (a) with trapezoidal shaper impulse response (b) and the output response of the system (c).

time, it is convenient to use a recursive convolution algorithm. The recursive form of moving average system is given as

$p(n)=\sum_{i=0}^{n} \nu(i)-\nu(i-l)$

or

$p(n)=p(n-1)+\nu(n)-\nu(n-l), \quad n \geq 0$,

where $\nu(n)$ is the prompt sample at time $n$ and $\nu(n-l)$ is a sample at a time that is delayed by $l$ relative to $n$. We will denote $l$ as the length of the convolution function.

The initial conditions that are imposed will determine the offset of the output signal. It is usually required that the offset be zero, so that the initial condition can be defined as

$\nu(n)=0, \quad n<0$.

Further, we assume that the same initial condition is met for the recursive algorithms to be discussed later.

The next step is to define a recursive algorithm for convolution using a truncated ramp function. Again it is assumed that the slope of the ramp is equal to unity. Under these circumstances the recursive form of the convolution can be written as

$r(n) \sum_{i=0}^{n}\left\{\sum_{j=0}^{l} \nu(j)-\nu(j-k)-\nu\left(i-k^{\prime}\right) k\right\}$

or

$r(n)=r(n-1)+p(n)-\nu\left(n-k^{\prime}\right) k, \quad n \geq 0$

Here $p(n)$ represents a moving average (Eq. (22)) with length $k$, and $k^{\prime}$ is a delay parameter. Three different impulse responses can be obtained depending upon the choice of value of $k^{\prime}$, as shown in Fig. 4. For the trapezoidal shaping discussed above it is necessary that $k^{\prime}$ be set equal to $k$.

Recursive convolution algorithms using higher order polynomial truncated functions can be constructed in fashion similar to that described for the truncated ramp case. For instance, a convolution with a trun-

a)

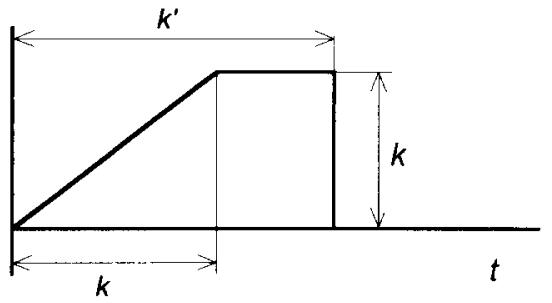

b)

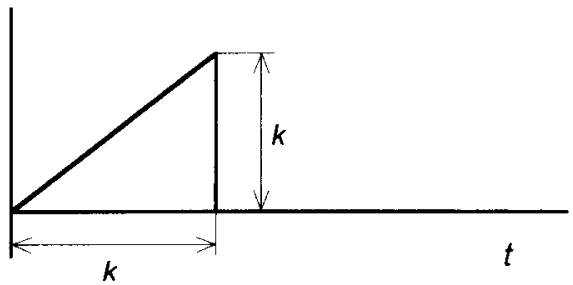

c)

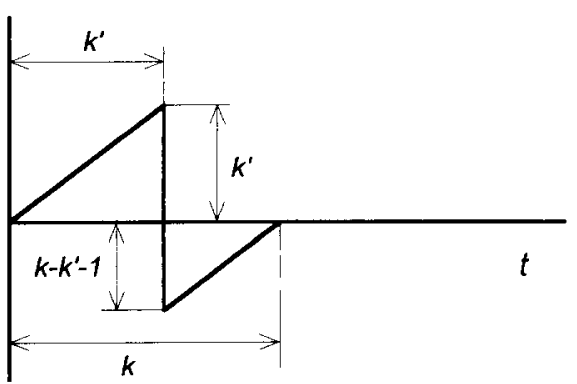

Fig. 4. Impulse response of a truncated ramp system at different values of the delay parameter $k^{\prime}$. (a) $k^{\prime}>k$, (b) $k^{\prime}=k$, (c) $k^{\prime}<k$. 
cated $\left(k^{2}+k\right) / 2$ function can be written in recursive form as

$$
\begin{aligned}
u(n)= & \sum_{l=0}^{n}\left\{\sum_{l=0}^{l}\left\{\sum_{j=0}^{l} \nu(j)-\nu(j-k)-\nu\left(i-k^{\prime}\right) k\right\}\right. \\
& \left.-\nu(l-k) \frac{k^{2}+k}{2}\right\}
\end{aligned}
$$

or

$u(n)=u(n-1)+r(n)-\nu(n-k) \frac{k^{2}+k}{2}, \quad n \geqslant 0$,

where $r(n)$ is truncated ramp convolution with length $k$ (Eq. (24)). Convolution with higher order polynomial functions requires operations with large integer numbers resulting in an increased complexity of the system. Thus, we will limit our considerations here to systems with rectangular and truncated ramp impulse responses.

The response of the trapezoidal shaper discussed previously in the continuous time domain can be written in the form of a recursive algorithm in the discrete time domain. It is assumed that the decay time constant of the sampled exponential signal is equal to $M$. The rise time of the trapezoidal shape is set to $k$ and the duration of the flat top of the trapezoidal shape is equal to $m=l-k$. From Eq. (21), the response of the system can be written as

$s(n)=r(n)+M p(n)+(k-M) p(n-k)-r(n-l)$.

From Eq. (22) and Eq. (24) the response of the system can be expressed in terms of sampled input signal

$$
\begin{aligned}
s(n)= & \sum_{i=0}^{n} \sum_{j=0}^{l}[\nu(j)-\nu(j-k)]-\nu(i-k) k \\
& +M \sum_{t=0}^{n}[\nu(i)-\nu(i-l)]
\end{aligned}
$$

$$
\begin{aligned}
& +(k-M) \sum_{l=0}^{n}[\nu(i-k)-\nu(i-l-k)] \\
& -\sum_{t=0}^{n} \sum_{J=0}^{l}[\nu(j-l)-\nu(j-k-l)] \\
& -\nu(i-k-l) k
\end{aligned}
$$

Let

$d^{k, l}(j)=\nu(j)-\nu(j-k)-\nu(j-l)+\nu(j-k-l)$.

The substitution of Eq. (28) into Eq. (27) yields

$s(n)=\sum_{i=0}^{n} \sum_{j=0}^{l} d^{k, l}(j)+d^{k, l}(i) M$.

The recursive form of Eq. (29) is given by

$s(n)=s(n-1)+p^{\prime}(n)+d^{k, l}(n) M, \quad n \geq 0$,

where

$p^{\prime}(n)=p^{\prime}(n-1)+d^{k, l}(n), \quad n \geq 0$.

Eqs. (30) and (31) define a recursive algorithm for generating a symmetric trapezoidal shape from a sampled exponential input signal. When $k=l$ the system response results in a symmetric triangular shape. Using Eqs. (30) and (31), a simple hardware configuration can be assembled using only a small number of standard digital pulse processing building blocks. A simplified block diagram of the trapezoidal shaper is depicted in Fig. 5.

Three programmable delay pipelines [9] are used for providing the necessary time shift of the sampled input signal. In subsequent steps, the signal passes through arithmetic units according to the algorithm described by Eqs. (30) and (31). All building blocks are integer arithmetic devices using signed variables. It should be noted that the digital processor passes just one digital value per processed pulse to the multichannel analyzer corresponding to the peak value of the shaped pulse. The approach is an extension of that employed in our previous work [8] where a similar

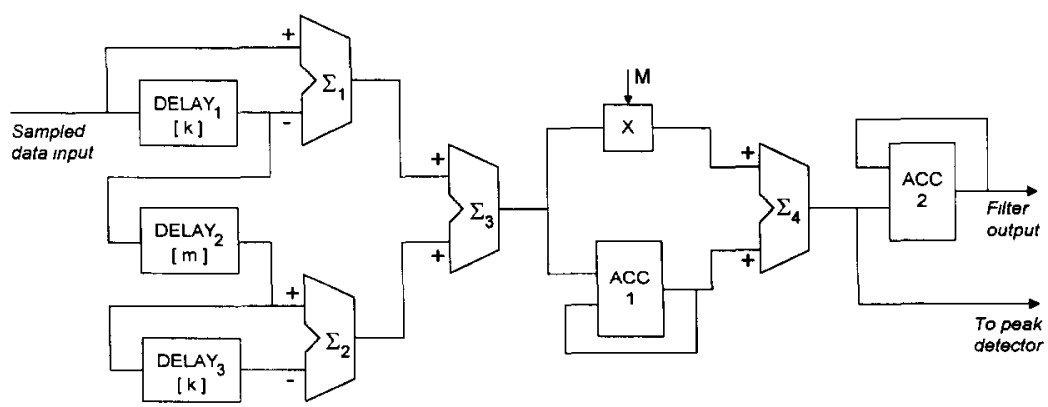

Fig. 5. Block diagram of digital trapezoidal/triangular shaping system. The building blocks are as follows: $\Sigma-$ adder/subtracter; ACC - accumulator, $\mathrm{X}$ - multiplier; DELAY - delay pipeline. 
threshold-free approach for detecting peaks has been described. Peaks are detected by monitoring sign changes in the result of the addition that precedes the final accumulation. When the noise level is high or when the sampling ADC misses some codes, measures need to be taken to avoid erroneous peak detection, especially in the case of a flat-topped pulse. Details of the peak detection process are given in refs. $[8,10,11]$.

\section{Other digital recursive algorithms}

The recursive algorithms described in previous sections allow a variety of different shapes to be synthesized in real time. When the time constant of the exponential input signal is comparable to the length of the convolution function the methodology described for the trapezoidal signal can be applied but with
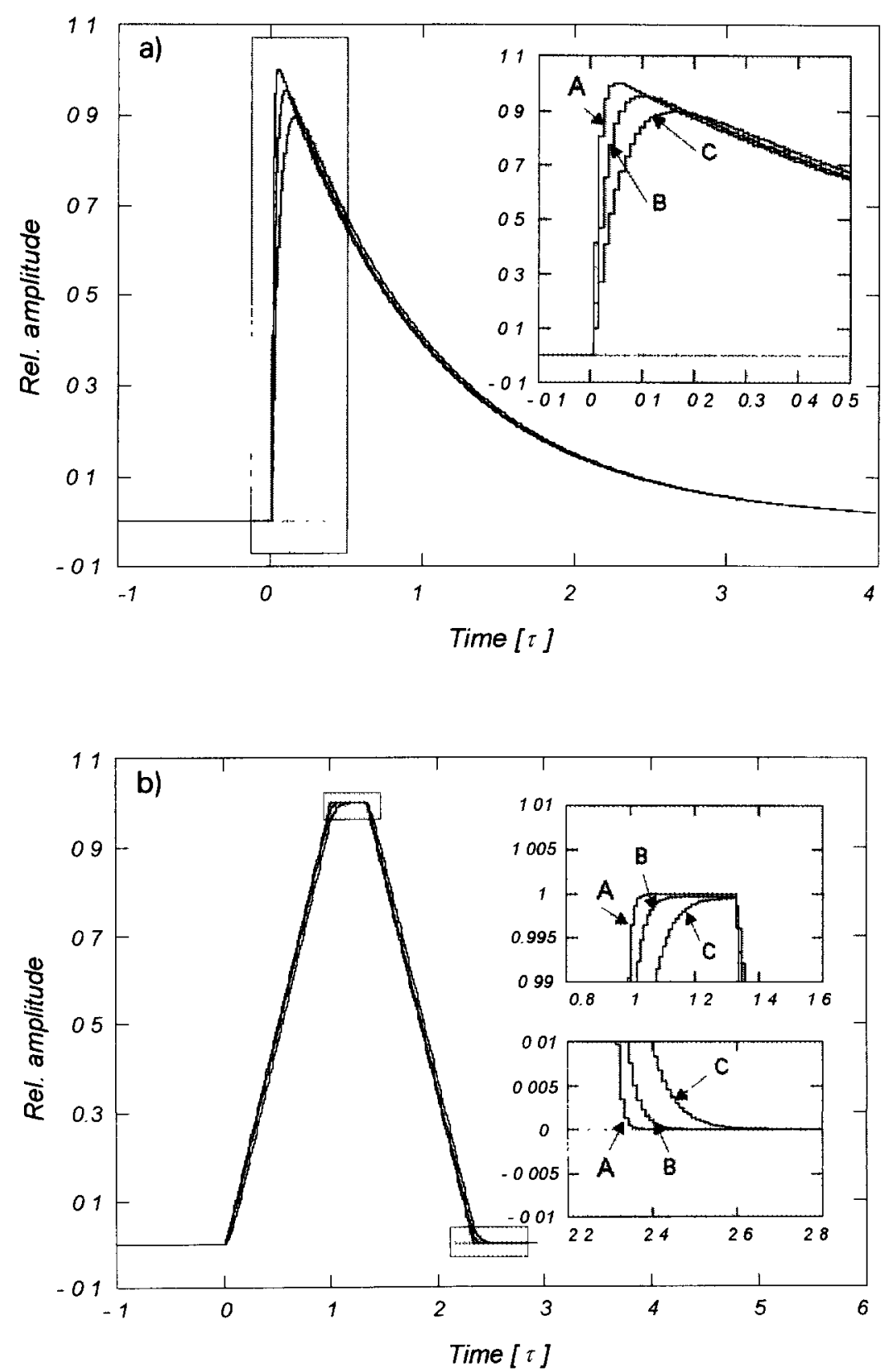

Fig. 6. Exponential pulses (see text) with different rise time (a) and the corresponding trapezoidal pulse shapes (b). 
Table 1

Digital recursive algorithms and the corresponding output responses to a step input signal

\begin{tabular}{|c|c|c|c|}
\hline \multirow[t]{3}{*}{$\underset{\forall(n)}{\text { INPUT }}$} & $\begin{array}{l}\text { RECURSIVE } \\
\text { ALGORITHM }\end{array}$ & $\begin{array}{c}\text { DELAY } \\
l\end{array}$ & $\begin{array}{l}\text { PULSE } \\
\text { SHAPE }\end{array}$ \\
\hline & $\begin{aligned} s(n)= & s(n-1)+v(n) \\
& -2 v(n-k)+v(n-2 k)\end{aligned}$ & & \\
\hline & $\begin{aligned} s(n)= & s(n-l)+v(n)-v(n-k) \\
& -v(n-l)+v(n-l-k)\end{aligned}$ & $l=k+m$ & \\
\hline & $\begin{array}{l}p(n)=p(n-l)+v(n)-v(n-l) \\
s(n)=s(n-l)+p(n)-v(n-k) l\end{array}$ & $i=2 k+I$ & \\
\hline & $\begin{aligned} & p(n)= p(n-l)+v(n)-v(n-k) \\
&+v(n-l)-v(n-l-k) \\
& s(n)= s(n-l)+p(n)-l v(n-k) \\
&+v(n-l)] k-v(n-l)+v(n-l-k)\end{aligned}$ & $l=k+m$ & $2 x+n$ \\
\hline
\end{tabular}

significantly different outcome. With the proper choice of parameters, finite cusp-like and flat-topped cusp-like shapes can be obtained. Optimization of the shapes often requires a solution of transcendental equations and the resulting pulse shape will be asymmetric. In this section, however, we will discuss a different case for which the input signal has a long decay constant and can be approximated as a step function. This circumstance holds when the output signal from a charge sensitive preamplifier is directly digitized [12].

First, consider the case of a step input. Using a rectangular convolving function, trapezoidal or triangular responses can be obtained. Cusp-like or truncated cusp-like pulse shapes can be synthesized using the recursive algorithms given by Eq. (24). Table 1 summarizes the recursive algorithms and corresponding pulse shapes. Because of the bipolar impulse responses of the systems shown in Table 1 , the amplitude of the shaped pulse is independent of any dc offset of the step input signal. Thus, the technique is directly applicable to the signal from a reset type preamplifier as well as that from a conventional resistive feedback preamplifier.

\section{Simulations and preliminary experimental tests}

\subsection{Calculated response}

In the previous sections we considered the rise time of the exponential pulse to be very short. In reality, however, the rise time of the signals is finite and depends on variety of factors: finite rise time of the analog circuits, CR networks in the path of the signal, finite charge collection time, etc.

We have therefore investigated the effect of finite rise time on the trapezoidal shaping steps that have, to this point, been illustrated for input pulses of zero rise time. The assumed input pulse shape was modified to be of the form

$\nu(t)=\frac{l}{\tau-\theta}\left(\mathrm{e}^{-t / \tau}-\mathrm{e}^{-t / \theta}\right)$,

where $\theta$ now accounts for the finite rise time of the pulse. In Fig. 6a, three input pulses (A, B, and C) are shown with values for $\tau / \theta$ of $0.01,0.025$ and 0.05 , respectively. In each case, the total charge (area under $\nu(t))$ has been kept constant. The rise time of the trapezoid is equal to decay time constant of the exponential signal. The amplitude of the output from trapezoidal shaper is given in units $\tau^{2}$. The flat top of the trapezoidal pulses in Fig. 6b has a duration of $\frac{1}{3}$ of the decay time constant $\tau$. The highlighted areas show the parts of the trapezoid which are affected the most by the variations in the rise time of the exponential pulse. Note that the maximum amplitude of the shaped output is the same for all three cases or, in other words, the output is free of the effects of ballistic deficit over this range of variation of the input rise time. The advantage of trapezoidal shaping in avoiding resolution loss due to ballistic deficit in germanium spectrometers has been previously recognized $[1,6,7]$. The time duration of the flat top must be long enough to accommodate the rise time variations, but should be made no longer than necessary to minimize the total pulse length and consequent effects of pile-up. One feature of the
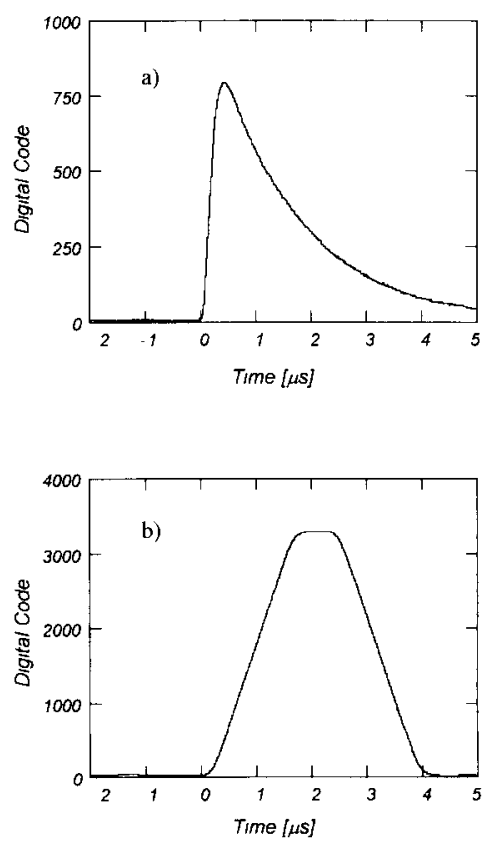

Fig. 7. Sampled input pulse (a) and corresponding trapezoidal shape (b). The decay time constant is $1.5 \mu \mathrm{s}$ and the flat top duration of the trapezoidal shape is $800 \mathrm{~ns}$. 
Table 2

Energy resolution of the digital trapezoidal/triangular shaper at different settings, and the energy resolution data which were obtained using a quasi-Gaussian shaper at the same measurement conditions

\begin{tabular}{|c|c|c|c|c|c|c|}
\hline $\begin{array}{l}\text { PULSE } \\
\text { SHAPE }\end{array}$ & \multicolumn{3}{|c|}{$\begin{array}{l}\text { HPGe DETECTOR } \\
\left({ }^{\infty} \mathrm{C} 0-133 \mathrm{MeV}\right)\end{array}$} & \multicolumn{3}{|c|}{$\begin{array}{c}\text { X-RAY DETECTOR } \\
\left({ }^{241} \mathrm{Am}-594 \mathrm{keV}\right)\end{array}$} \\
\hline & $\begin{array}{l}T_{F}^{*} \\
f \mu s\}\end{array}$ & $\begin{array}{l}T_{\mathrm{w}} \\
{[\mu \mathrm{\mu}]} \\
\end{array}$ & $\begin{array}{c}\text { FWHM } \\
{[\mathrm{keV}]}\end{array}$ & $\begin{array}{l}T_{F}^{*} \\
{[\mu \mathrm{w} J} \\
\end{array}$ & $\begin{array}{l}T_{w} \\
\langle\mu s] \\
\end{array}$ & $\begin{array}{c}F W H M \\
{[\mathrm{keV}]}\end{array}$ \\
\hline & $o$ & 68 & 1735 & 0 & 72 & 112 \\
\hline & 02 & 70 & 928 & 02 & 74 & 112 \\
\hline & 04 & 72 & 243 & 04 & 76 & 112 \\
\hline & 06 & 74 & 241 & 06 & 78 & 113 \\
\hline & 06 & 32 & 340 & 0 & 38 & 131 \\
\hline \multirow{3}{*}{$T_{\rho}$} & $\begin{array}{l}T_{p} \\
{[\boldsymbol{\mu}\rfloor}\end{array}$ & $\begin{array}{l}T_{w}{ }^{+} \\
\langle\boldsymbol{\mu} s\rfloor\end{array}$ & $\begin{array}{c}\text { FWHM } \\
{[\mathrm{keV}]}\end{array}$ & $\begin{array}{l}T_{p} \\
i \mu r j\end{array}$ & $\begin{array}{l}T_{w}{ }^{\dagger} \\
{[\mu \leqslant s]}\end{array}$ & $\begin{array}{c}\text { FWHM } \\
{[\mathrm{keVI}]}\end{array}$ \\
\hline & 2 & 55 & 574 & 2 & 55 & 138 \\
\hline & 4 & $H$ & 241 & 4 & $n$ & 118 \\
\hline \multirow[t]{2}{*}{$T_{W}$} & 6 & 16 & 210 & - & -- & \\
\hline & 12 & 30 & 198 & -- & $\ldots$ & -. \\
\hline
\end{tabular}

* This entry is the delay $m=k-1$ (see Eq. (28)-(31)).

Width of the pulse at $2 \%$ of the amplitude.

present digital approach to shaping is that the flat top duration can be easily adjusted to suit the application by a simple change in choice of delay settings.

\subsection{Experimental measurements}

In order to test the techniques for synthesis of triangular and trapezoidal shapes, a prototype system was assembled that implements the algorithms developed earlier in this paper in a personal computer (PC) on a pulse-by-pulse basis. While this configuration is not the full hardware implementation we envision for the finished system (such as illustrated in Fig. 5), it is capable of testing the principles at data rates that are limited by data transfer and processing time in the PC. A full hardware implementation would impose no rate limitations, and maximum throughput would be set only by pile-up considerations.

In our prototype, the input pulses were obtained from the fast output of a spectroscopy amplifier (Ortec 673) [13]. This signal has a rise time $(10-90 \%)$ of approximately $220 \mathrm{~ns}$ and an exponential decay time constant of either 1.5 or $3.5 \mu$ s (these are approximate values and depend on pole-zero adjustment). This pulse was sampled using a 10-bit ADC (AD9020) operating at $50 \mathrm{MHz}$. Time slices of up to $20 \mu \mathrm{s}$ length were temporarily stored for each pulse in a FIFO buffer and then transmitted to the PC for implementation of the recursive shaping algorithms. The output of the shaper was then stored in a multichannel spectrum based on a trigger provided by the peak sensing algorithms similar to those described in ref. [8]. For comparison purpose, the quasi-Gaussian analog output of the same spectroscopy amplifier was also supplied to a conventional multichannel analyzer.

A typical time slice including the input signal and the corresponding trapezoidal shape output pulse is shown in Fig. 7. A digital gain of 4 was used so that the amplitude of the shaped signal is approximately 4 times greater than the amplitude of the exponential signal.

We used two detectors to evaluate the performance of the digital shaping system. The first was a p-type HPGe with $30 \%$ relative efficiency, while the second was a $\mathrm{p}$-i-n photodiode used as X-ray detector. The counting rate was kept low (less than $1 \mathrm{kcps}$ ) in order to ensure that pile-up would have no significant effect on the recorded spectra. Table 2 summarizes the obtained results.

The data presented in Table 2 shows the superiority of the digital trapezoidal/triangular shaping system compared with the quasi-Gaussian shaper. Specifically, the digital system allows a shorter shaping time to be chosen at equivalent energy resolution, compared with the quasi-Gaussian shaper. This advantage will be most important at high counting rates where pile-up is a major consideration. By increasing the duration of the flat-topped part of the trapezoid, the effects of ballistic deficit are eliminated. It is also evident that, in the case of a detector with a very short charge collection time (the X-ray detector), the energy resolution is independent of the duration of the flat part of the pulse shape which is in good correspondence with theoretical predictions $[1,3]$. In obtaining these results we have found that proper adjustment of pole-zero is critical to provide an input pulse with a single exponential decay.

The results shown in Table 2 indicate a distinct advantage of the prototype digital shaper over the analog system used for comparison purposes. However, several words of caution are in order in interpreting these preliminary results. The system used to obtain these data operated in quasi-real time, and was not a true on-line processor of the type illustrated in Fig. 5. While we are reasonably confident that such a system could produce similar results, that capability has not yet been demonstrated. Also, the analog shaping amplifier used for comparison purposes is a common commercially available unit, but may not represent the ultimate performance that could be obtained with a custom designed analog pulse processing system.

\section{Conclusion}

Recursive algorithms for the digital synthesis of trapezoidal and triangular pulse shapes have been de- 
veloped. Priority was given to real time applicability of the shaping steps and their simplicity. The technique offers flexibility through simple software adjustment of the shape parameters depending on the characteristics of the detector. Particularly, it has been demonstrated that by varying (digitally) just one parameter (the duration of the flat top of the trapezoid) an effective elimination of the effects of ballistic deficit from an HPGe detector can be achieved.

\section{References}

[1] V. Radeka, IEEE Trans. Nucl. Sci. NS-15 (1968) 455.

[2] P.W. Nicholson, Nuclear Electronics (Wiley, New York, 1974).

[3] F.S. Gouldıng, Nucl. Instr. and Meth. 100 (1972) 493.
[4] F.S. Goulding, D.A. Landıs and N.W. Madden, IEEE Trans. Nucl. Sci. NS-30 (1983) 301.

[5] K. Debertin and R.G. Helmer, Gamma- and X-ray Spectrometry with Semiconductor Detectors (North-Holland, Amsterdam, 1988).

[6] F.S. Goulding et al, presented at IEEE NSS, San Francisco, Nov. 1993.

[7] S.M. Hinshaw and D.A. Landis, IEEE Trans. Nucl. Sc1. NS-35 (1990) 374.

[8] V. Jordanov and G.F. Knoll, IEEE Trans. Nucl Scı. NS-40 (1993) 764.

[9] V. Jordanov, EDN 37 (Nov. 12, 1992) 200.

[10] F. Hilsenrath, H.D. Voss and J.C. Bakke, IEEE Trans. Nucl. Sci. NS-32 (1985) 145.

[11] T.H. Wilmshurst, Signal Recovery from Norse in Electronic Instrumentation, 2nd ed. (Adam Hılger, Bristol, 1990).

[12] T. Lakatos, Nucl. Instr. and Meth. B 62 (1991) 289.

[13] Ortec 673 spectroscopy amplifier and gated integrator manual (Ortec, 1984). 